\title{
Elite Haplotypes of a Protein Kinase Gene TaSnRK2.3 Associated with Important Agronomic Traits in Common Wheat
}

\begin{abstract}
Lili Miao't, Xinguo Mao ${ }^{2,3+}$, Jingyi Wang ${ }^{2}$, Zicheng $\mathrm{Liu}^{2}$, Bin Zhang ${ }^{2}$, Weiyu $\mathrm{Li}^{2}$, Xiaoping Chang ${ }^{2}$, Matthew Reynolds ${ }^{4}$, Zhenhua Wang ${ }^{1 *}$ and Ruilian Jing ${ }^{2 *}$
\end{abstract}

${ }^{1}$ College of Agronomy, Northeast Agricultural University, Harbin, China, ${ }^{2}$ National Key Facility for Crop Gene Resources and Genetic Improvement, Institute of Crop Science, Chinese Academy of Agricultural Sciences, Beijing, China, ${ }^{3}$ College of Life Science and Technology, Gansu Agricultural University, Lanzhou, China, ${ }^{4}$ International Maize and Wheat Improvement Center, Texcoco, Mexico

OPEN ACCESS

Edited by:

Daniel Pinero,

National Autonomous University

of Mexico, Mexico

Reviewed by:

Yong $X u$,

National Engineering Research Center

for Vegetables, China

Guijun Yan,

University of Western Australia,

Australia

*Correspondence:

Zhenhua Wang

zhenhuawang_2006@163.com

Ruilian Jing

jingruilian@caas.cn

†These authors have contributed equally to this work.

Specialty section:

This article was submitted to Plant Genetics and Genomics,

a section of the journal

Frontiers in Plant Science

Received: 03 November 2016 Accepted: 01 March 2017 Published: 28 March 2017

Citation:

Miao L, Mao X, Wang J, Liu Z, Zhang B, Li W, Chang $X$, Reynolds $M$, Wang $Z$ and Jing $R$ (2017) Elite Haplotypes of a Protein Kinase Gene TaSnRK2.3 Associated with Important Agronomic Traits in Common Wheat.

Front. Plant Sci. 8:368. doi: 10.3389/fpls.2017.00368
Plant-specific protein kinase SnRK2s play crucial roles in response to various environmental stimuli. TaSnRK2.3, a SnRK2 member, was involved in the response to multiple abiotic stresses in wheat. To facilitate the use of TaSnRK2.3 in wheat breeding, the three genomic sequences of TaSnRK2.3, originating from the $\mathrm{A}, \mathrm{B}$, and $D$ genomes of hexaploid wheat, were obtained. Sequence polymorphism assays showing 4 and 10 variations were detected at TaSnRK2.3-1A and at TaSnRK2.3-1B, respectively, yet no variation was identified at TaSnRK2.3-1D. Three haplotypes for $A$ genome, and two main haplotypes for B genome of TaSnRK2.3 were identified in 32 genotypes. Functional markers (2.3AM1, 2.3AM2, 2.3BM1, 2.3BM2) were successfully developed to distinguish different haplotypes. Association analysis was performed with the general linear model in TASSEL 2.1. The results showed that both TaSnRK2.3$1 A$ and TaSnRK2.3-1B were significantly associated with plant height $(\mathrm{PH})$, length of peduncle and penultimate node, as well as 1,000-grain weight (TGW) under different environments. Additionally, TaSnRK2.3-1B was significantly associated with stem water-soluble carbohydrates at flowering and mid-grain filling stages. Hap-1A-1 had higher TGW and lower PH; Hap-1B-1 had higher TGW and stem water-soluble carbohydrates, as well as lower $\mathrm{PH}$, thus the two haplotypes were considered as elite haplotypes. Geographic distribution and allelic frequencies indicated that the two preferred haplotypes Hap-1A-1 and Hap-1B-1 were positively selected in the process of Chinese wheat breeding. These results could be valuable for genetic improvement and germplasm enhancement using molecular marker assisted selection in wheat breeding.

Keywords: TaSnRK2.3, association analysis, haplotype, functional marker, stem water-soluble carbohydrates

\section{INTRODUCTION}

During the processes of growth and development, plants are vulnerable to various kinds of abiotic stresses, including drought, high salinity, and extreme temperature because of their immobility. To survive, plants have evolved a number of ways to cope with versatile environmental stresses. Protein kinases and phosphatases, are major components of intracellular signal transduction, and play important roles in multi-environmental stress responses (Hong et al., 1997). 
Diverse stress-inducible protein kinase families mainly include mitogen-activated protein kinase (Wrzaczek and Hirt, 2001), calcium-dependent protein kinase (Ludwig et al., 2004), and sucrose non-fermenting 1 (SNF1)-related protein kinase (SnRK). Most of them are activated by abscisic acid (ABA) or environmental stimuli. SNF1 protein kinase in yeast, AMPactivated protein kinase in mammals, and plant SnRK (especially SnRK1) protein are highly conserved and play pivotal roles in growth and metabolic response to cellular stresses as energy sensors.

The SnRK family is classified into three subfamilies in plants, i.e., SnRK1, SnRK2, and SnRK3, based on sequence similarity, gene structures, and expression patterns. SnRK2 is a relatively small plant-specific subfamily, encoding serine/threonine kinases. The SnRK2s contain two typical domains, viz., an $\mathrm{N}$-terminal catalytic domain which plays an important role in kinase activation, and a regulatory $\mathrm{C}$-terminal region involved in protein-protein interactions and possibly in ABA signaling (Huang et al., 1996; Vlad et al., 2009). The SnRK2s were further divided into three subclasses in phylogeny according to their varied activation patterns in response to ABA (Kobayashi et al., 2004). Among them, subclass III was strongly induced by ABA, weakly for subclass II, and no activation for subclass I. Accumulated evidence indicated that SnRK2 is a merging point of ABA-dependent and ABA-independent pathways in abiotic stress responses and developmental processes in plants (Fujii et al., 2011; Kulik et al., 2011). Our target gene TaSnRK2.3 belongs to subclass II in common wheat (Triticum aestivum L.) (Zhang et al., 2016). In previous research, we cloned and characterized $\operatorname{TaSnRK2.3}$ in wheat, its hetero-expression resulted in improved tolerances to multiple abiotic stresses (Tian et al., 2013).

Wheat is one of the most important cereal crops worldwide, while its growth and development is severely influenced by abiotic stresses, resulting in significant reduction in grain yield. In the scenario of climate change, mining and utilization of key genes conferring tolerances to abiotic stress is regarded as an effective way to ensure a high and stable yield in wheat. However, common wheat is a hexaploid species (AABBDD) with a very large and complex genome $\left(17.9 \times 10^{9} \mathrm{bp}\right)$, enriched in abundant repeat sequences (about 86\%) (Varshney et al., 2006), hence it is still a serious challenge to directly isolate a gene and further decipher its function at the molecular level, although three genome drafts of diploid and hexaploid wheat have been constructed (Jia et al., 2013; Ling et al., 2013; International Wheat Genome Sequencing Consortium [IWGSC], 2014). Marker assistant selection (MAS) based on elite allele pyramiding is considered a potential approach to wheat improvement for complex traits. As the third generation molecular marker, single nucleotide polymorphism (SNP) featured with high abundance and stability, cost efficiency, and high-throughput scoring, has been widely used in plant heredity and breeding (Collard and Mackill, 2008; Wang et al., 2015). With the development of high density SNPs and other molecular markers, association analysis has become an efficient tool to identify the relationship between markers or polymorphism sites of target genes and traits, and has been successfully used in Arabidopsis thaliana (Nemri et al., 2010), rice (Agrama et al., 2007), maize (Thornsberry et al., 2001; Li et al., 2010), and wheat (Zhang et al., 2015; Li et al., 2016). Mining causative molecular polymorphisms and developing functional markers are fundamental to stacking superior alleles of key genes in genetic improvement of crops using MAS methods (Wang et al., 2016).

To facilitate utilization of TaSnRK2.3 in wheat molecular breeding by MAS, our research mainly concentrated on: (i) isolating and characterizing three genomic sequences of TaSnRK2.3 in common wheat, (ii) identifying polymorphism sites and developing functional markers in TaSnRK2.3-1A/1B, (iii) identifying favorable allelic variations and haplotypes for TaSnRK2.3-1A/1B by association analysis, (iv) revealing the distribution of preferred genotypes in varieties released in different years and geographical environments in China. The results can offer valuable information for wheat improvement.

\section{MATERIALS AND METHODS}

\section{Plant Materials and Measurement of Agronomic Traits and Stem Water-Soluble Carbohydrates}

Common wheat cultivar Hanxuan 10 with remarkable tolerance to drought stress was used for genomic sequence isolation of TaSnRK2.3 and gene structure analysis. Twelve accessions of various wheat species, including three A genome accessions (Triticum urartu) (UR204, UR206, and UR207), three S genome accessions (Aegilops speltoides, the putative $\mathrm{B}$ genome donor) (Y2003, Y2033, and Y2017), three D genome accessions (Ae. tauschii) (Y125, Y225, and AE38), and three AB genome accessions (T. dicoccoide) (DS1, PS5 and PS9) were selected for target fragment isolation and genomic origin identification.

Thirty-two accessions/genotypes with wide variation screened by SSR markers, were initially chosen to re-sequence for polymorphism analysis. Three hexaploid wheat germplasm populations were selected for different research purposes. Population 1 (262 accessions/genotypes) was firstly employed for association analysis. The accessions were mainly released in the Northern winter wheat and Yellow and Huai River valley facultative wheat zones (Zhang et al., 2015). Population 2 (157 landraces/genotypes) and Population 3 (348 modern cultivars/genotypes) were used to determine temporal haplotypes and analyze geographic distribution aiming to functionally validate TaSnRK2.3-1A/1B markers. Population 2 was mainly from the Chinese wheat mini-core collection representing more than $70 \%$ of the genetic diversity of the total Chinese germplasm collection; Population 3 came from the Chinese wheat core collection (Hao et al., 2008; Hao et al., 2011). The two populations (2 and 3) including genotypes from all the 10 Chinese wheat production zones were selected from 23, 705 accessions released or collected in China (Zhang et al., 2002; Dong et al., 2003; Hao et al., 2008).

Population 1 was planted in 10 environments (year $\times$ site $\times$ water regime combinations) including Changping $\left(116^{\circ} 13^{\prime} \mathrm{E}, 40^{\circ} 13^{\prime} \mathrm{N}\right)$, Beijing, in 2010 and 2012, and Shunyi 
$\left(116^{\circ} 56^{\prime} \mathrm{E}, 40^{\circ} 23^{\prime} \mathrm{N}\right)$, Beijing, in 2010, 2011, and 2012. The field experiments were grown under well-watered (WW) and drought-stressed (DS) regimes. The WW plots were irrigated with $750 \mathrm{~m}^{3} \mathrm{ha}^{-1}(75 \mathrm{~mm})$ at each of pre-overwintering, booting, flowering, and grain filling stages (total $300 \mathrm{~mm}$ applied as irrigation), while DS plots were rain-fed. The rainfall during the growing seasons were $131 \mathrm{~mm}$ in 2010, $180 \mathrm{~mm}$ in 2011, and $158 \mathrm{~mm}$ in 2012. Measured agronomic traits included 1,000-grain weight (TGW), plant height $(\mathrm{PH})$, peduncle length (PLE), length of penultimate node (LPN), spike length, number of spikes per plant, total number of spikelets per spike, number of sterile spikelets per spike, and grain number per spike.

Stem water-soluble carbohydrates (SWSC) are an important carbon source for grain filling in wheat. They are mainly composed of fructans, sucrose, glucose, and fructose, with the main reserve as fructans at the late stage of WSC accumulation (Ruuska et al., 2006). We obtained SWSC data of Population 1 under WW and DS conditions. SWSC were measured by nearinfrared reflectance spectroscopy (MAP multi-purpose FT-NIR analyzer) as previously described (Wang et al., 2011). Five main stems were cut $1 \mathrm{~cm}$ above the soil surface at the flowering, midgrain filling (14 days after flowering), and maturity stages. Leaf blades were removed from samples, and stem samples were cut into two parts, namely, peduncle, and the lower internodes except for peduncle. The WSC was determined for peduncle, lower internode and total stem, using different near-infrared reflectance spectroscopy (NIRS) regression models, which were developed for quantitative determination of WSC using modeling samples of $150 \mathrm{DH}$ (Hanxuan $10 \times$ Lumai 14) lines (Wang et al., 2011).

\section{Isolation and Sequencing of Genomic Region Surrounding TaSnRK2.3}

For each accession, genomic DNA was extracted from young leaves by cetyltrimethylammonium bromide (CTAB) method (Stewart and Via, 1993). Based on known cDNA information, a primer pair GF/R (Table 1) was obtained to amplify the genome sequence of TaSnRK2.3. TransStart Fast Pfu DNA polymerase was used in PCR amplification (TransGen, Inc). PCR products were extracted and cloned into $p E A S Y$-Blunt vector, 24 clones for each sample were randomly selected for sequencing by DNA Analyzer 3730XL. To get the whole sequence of TaSnRK2.3, both M13 and three overlapping primers (Table 1) were used for sequence walking. So the sequence of each clone was obtained by assembling five overlapping sequences with the SeqMan program in DNAStar. The genomic origin of each of the sequences were confirmed by comparing them with that from diploid and tetraploid species based on clustalW analysis with the MegAlign program.

\section{Functional Marker Development}

A total of four functional markers (2.3AM1, 2.3AM2, 2.3BM1, 2.3BM2) were developed based on four selected polymorphism sites, in order of $1898 \mathrm{bp}(\mathrm{C} / \mathrm{T})$ and $2905 \mathrm{bp}(\mathrm{A} / \mathrm{G})$ of $\mathrm{A}$ genome; $2153 \mathrm{bp}(\mathrm{C} / \mathrm{T})$ and $2638 \mathrm{bp}(\mathrm{C} / \mathrm{G})$ of $\mathrm{B}$ genome. These markers were designed with a specific mismatch in the primer to introduce a restriction enzyme recognition site using an available program dCAPS Finder $2.0^{1}$. Basically, the four systems of PCR and digestion were similar. Genotyping was performed by two rounds of PCR. Firstly, genome specific primer pairs were used to amplify fragments from chromosome $1 \mathrm{~A} / 1 \mathrm{~B}$ in all accessions. The second round of PCR was performed as follows: the first round of PCR product was diluted 50 times, then taking $1 \mu \mathrm{l}$ as template for the second round of PCR. The annealing temperatures and extension time were set depending on primer pairs and expected PCR product lengths. The PCR products were resolved by electrophoresis in $4 \%$ agarose gel after digestion with corresponding restriction enzymes. The primers were listed in Table 1.

${ }^{1}$ http://helix.wustl.edu/dcaps/dcaps.html

TABLE 1 | Primers used for genomic fragment isolation, sequencing and marker development.

\begin{tabular}{|c|c|c|}
\hline Primer set & Nucleotide sequence $\left(5^{\prime}\right.$ to $\left.3^{\prime}\right)$ & Experimental purpose \\
\hline GF & GTTTGCTCTGAGTTGGTGCTTC & \\
\hline GR & CAGTAATAATGTACACAGCGATAG & $A, B$, and D genomic fragment amplification \\
\hline M13F & TGTAAAACGACGGCCAGT & \\
\hline M13R & CAGGAAACAGCTATGACC & \\
\hline SeqF1 & ACTCGTTAGTCGTITGATAAGTT & \\
\hline SeqF2 & GGGCGATTCAGTGAAGAT & \\
\hline SeqR1 & CATGTGACATCTCAGAACCAT & Sequencing primers for TaSnRK2.3 \\
\hline AGF & TTCACAGTCGGTTCGTTCG & A genome specific target fragment amplification \\
\hline BGF & TAAGTACCGCTTATGACAATCTGTGGTT & B genome specific target fragment amplification \\
\hline AG-Hhal-F & TACAACATAGAACTITAGTAATGGACAGCG & \\
\hline AG-Hhal-R & TCACGCCGCAGGACCAA & Marker 2.3AM1 developed for SNP-1898 (C/T) \\
\hline AG-Xbal-F & CTTCAAGGAGCCCGAGACG & \\
\hline AG-Xbal-R & TTCTTGTTTCAGCAAACCGTACTC & Marker 2.3AM2 developed for SNP-2905 (A/G) \\
\hline BG-N1alll-F & AACTCTGATCTGAACACGAATGCA & \\
\hline BG-N1alll-R & GTGATCCTCTTCAGAGTICAGACAC & Marker 2.3BM1 development for SNP-2153 (C/T) \\
\hline BG-Avall-F & AAGGCAGAAAACTITGAATCATAAC & \\
\hline BG-Avall-R & TCCTCATCGCTCTGCAGCTCGGCCAGGTC & Marker 2.3BM2 development for SNP-2638 (C/G) \\
\hline
\end{tabular}




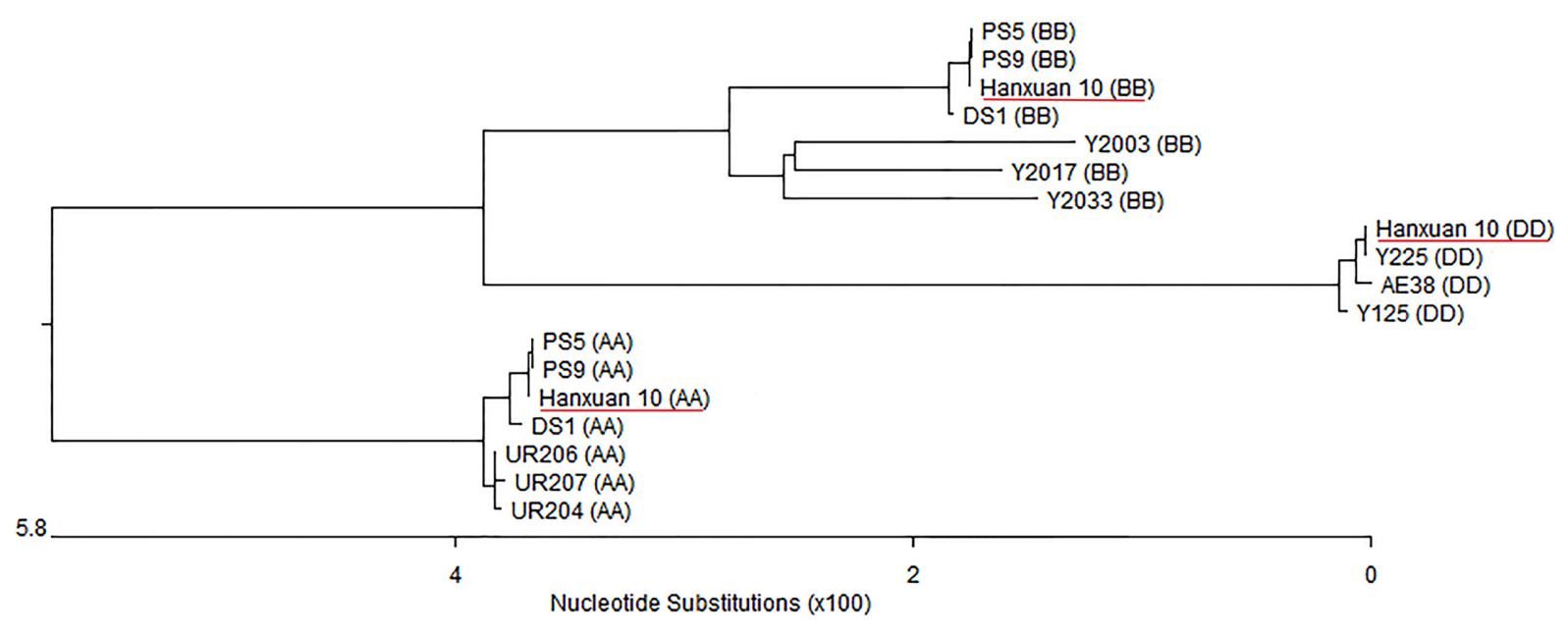

FIGURE 1 | Genomic origin identification of TaSnRK2.3s based on phylogenetic tree constructed with MegAlign program. AA, Triticum urartu; SS, Aegilops. speltoides (closely related to BB); DD, Ae. Tauschii; AABB, T. dicoccoide; AABBDD, hexaploid accession Hanxuan 10.

A

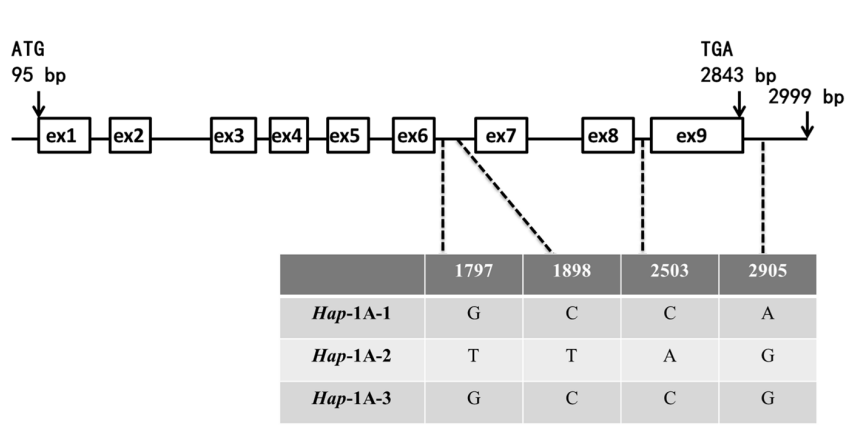

B

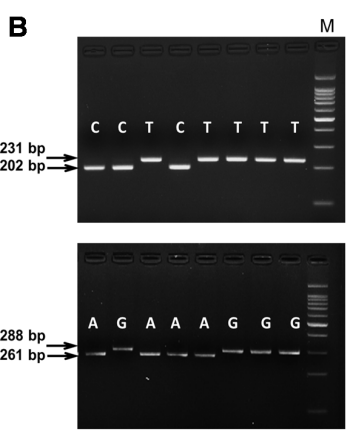

FIGURE 2 | Nucleotide polymorphisms and functional marker development of TaSnRK2.3-1A. (A) Four single nucleotide polymorphisms (SNPs) were detected in the non-exon regions of TaSnRK2.3-1A. (B) A dCAPS marker 2.3AM1 was developed based on SNP-1898 (C/T) in the upper segment. Digestion of the amplified 231 bp fragment with Hhal produced fragments of 202 bp/29 bp for accessions with SNP-1898C, yet unacted for accessions with SNP-1898T. Similarly, a CAPS marker 2.3AM2 from SNP-2905 (A/G) was also developed in the lower segment. M, 100 bp DNA Ladder.

\section{Association Analysis}

TASSEL 2.1 was used to identify significant associations between haplotypes and agronomic traits for Population 1. The general linear model (GLM) was performed using population structure Q matrix, which listed the estimated membership coefficients for each individual in each cluster. Associations were considered significant at $P<0.05$. Different effects of haplotype on traits were analyzed by one-way ANOVA using SPSS 16.0 software, and followed by the least significant difference (LSD) method at $P<0.05$ (even 0.01).

\section{RESULTS}

\section{Genetic Characterization of TaSnRK2.3}

Based on TaSnRK2.3 cDNA, three genomic sequences of TaSnRK2.3 were isolated from Hanxuan 10 using the primer pair GF/R flanking the open reading frame. The sequencing results indicated that the three types of sequences obtained were consistent with the previous results (Tian et al., 2013). By comparing different TaSnRK2.3 genomic sequences among the ploidy levels of different wheat species, the genomic origin was identified (Figure 1). Since TaSnRK2.3 was located on chromosome 1A, 1B, and 1D (Tian et al., 2013), we named the three copies TaSnRK2.3-1A, TaSnRK2.3-1B, and TaSnRK2.3-1D, respectively.

Multialignment (ClustalW) assays showed that TaSnRK2.3 consisted of nine exons and eight introns (Figures 2, 3). The fragment sizes of TaSnRK2.3-1A, TaSnRK2.3-1B, and TaSnRK2.3$1 D$ were $2,999,2,862$, and $2,817 \mathrm{bp}$, and their homologies ranged from 92.5 to $94.4 \%$.

\section{Sequence Polymorphism Assays and Marker Development}

As shown in Figure 2, for TaSnRK2.3-1A, polymorphic sites were only identified in non-exon regions in 32 accessions, and the four SNPs formed three haplotypes (Hap-1A-1, Hap-1A-2, and 

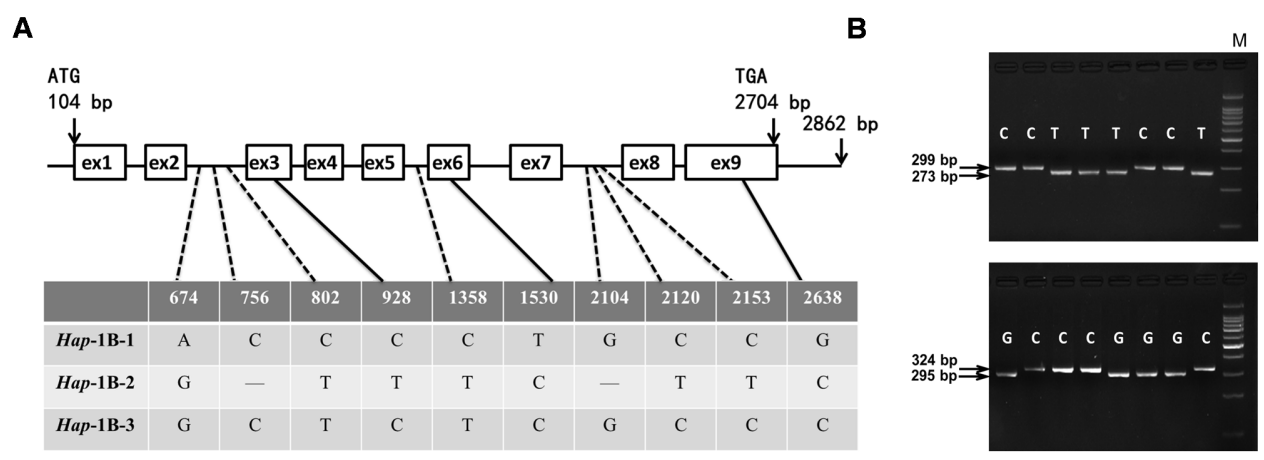

FIGURE 3 | Nucleotide polymorphisms and functional marker development of TaSnRK2.3-1B. (A) Eight SNPS and two InDels were detected in the coding region of TaSnRK2.3-1B. "-" indicates deletion. (B) CAPS marker 2.3BM1 was developed based on SNP-2153(C/T). Digestion of the amplified 299 bp fragment with N1all produced fragments of 273 bp/26 bp for accessions with SNP-2153T, and a single 299-bp band for accessions with SNP-2153C. Similarly, a dCAPS marker 2.3BM2 for SNP-2638(C/G) was developed. M, 100 bp DNA Ladder.

Hap-1A-3). Ten nucleotide variations (eight SNPs and two 1-bp InDels) were detected in the target fragment of TaSnRK2.3-1B in the 32 common wheat accessions (Figure 3), only SNP at $928 \mathrm{bp}$ $(\mathrm{C} / \mathrm{T})$ led to an amino acid change $(\mathrm{CCG} \rightarrow$ Pro, $\mathrm{TCG} \rightarrow$ Ser $)$. The 10 SNPs formed three haplotypes, i.e., Hap-1B-1, Hap-1B-2, and Hap-1B-3.

To distinguish the haplotypes of TaSnRK2.3-1A, two functional markers (2.3AM1 and 2.3AM2) were developed based on the chosen SNP sites. One was a HhaI cutting site at SNP-1898C, un-cut at SNP-1898T; the other was an XbaI cutting site at SNP-2905A, un-cut at SNP-2905G (Figure 2). In the same way, markers $2.3 \mathrm{BM} 1$ and $2.3 \mathrm{BM} 2$ for TaSnRK2.3-1B were successfully developed with restriction enzymes N1aIII and AvaII (Figure 3).

\section{Association Analysis of TaSnRK2.3-1A Haplotypes and Agronomic Traits}

For TaSnRK2.3-1A, Hap-1A-2 was a major haplotype accounting for $71.5 \%$ frequency in the natural population, followed by Hap$1 \mathrm{~A}-1$ with a frequency of $22.3 \%$, and Hap-1A-3 with the lowest percentage (6.2\%). Association analysis showed that the three haplotypes were significantly associated with PH, PLE, LPN, and TGW (Table 2). Hap-1A-1 had the lowest PH, PLE, and LPN among the three haplotypes in almost all the environments, with significant difference $(P<0.01)$ between Hap-1A-1 and the others. The TGW of Hap-1A-1/2 was higher than Hap-1A3 in 10 environments, and the differences were significant in seven environments $(P<0.01$ or 0.05$)$ (Figure 4). Therefore, Hap-1A-1 could be a superior allele for increasing TGW and reducing $\mathrm{PH}$.

\section{Association Analysis between Haplotypes of TaSnRK2.3-1B and Agronomic Traits}

For TaSnRK2.3-1B, Hap-1B-1 and Hap-1B-2 were two major haplotypes, accounting for 46.1 and $53.5 \%$ frequency. Hap-1B3 was a rare haplotype only presented in one accession, thus it was not included in subsequent statistical analysis. Significant associations were identified between TaSnRK2.3-1B haplotypes and agronomic traits, including PH, PLE, and LPN in all 10

TABLE 2 | TaSnRK2.3-1A haplotypes associated with agronomic traits in 10 environments.

\begin{tabular}{|c|c|c|c|c|c|c|c|c|}
\hline \multirow[t]{2}{*}{ Environments } & \multicolumn{2}{|c|}{ PH } & \multicolumn{2}{|c|}{ PLE } & \multicolumn{2}{|c|}{ LPN } & \multicolumn{2}{|c|}{ TGW } \\
\hline & $P$-value & PVE (\%) & $P$-value & PVE (\%) & $P$-value & PVE (\%) & $P$-value & PVE (\%) \\
\hline 2010-CP-WW & $2.58 \mathrm{E}-06^{* * *}$ & 13.56 & $9.11 \mathrm{E}-06^{* * *}$ & 12.17 & 3.33E-05*** & 10.75 & n.s. & - \\
\hline 2010-CP-DS & 8.77E-07*** & 14.76 & $1.31 \mathrm{E}-06^{* * *}$ & 14.31 & $0.0048^{* *}$ & 5.46 & $0.0343^{*}$ & 3.42 \\
\hline 2010-SY-WW & $1.29 \mathrm{E}-05^{* * *}$ & 11.83 & $1.17 \mathrm{E}-05^{* * *}$ & 11.93 & $1.40 \mathrm{E}-04^{* * *}$ & 9.22 & $0.0177^{*}$ & 4.10 \\
\hline 2010-SY-DS & $1.01 \mathrm{E}-06^{* * *}$ & 14.59 & $2.86 \mathrm{E}-05^{* * *}$ & 10.91 & $9.85 \mathrm{E}-07^{* * *}$ & 14.62 & $0.0302^{*}$ & 3.55 \\
\hline 2011-SY-WW & $4.08 \mathrm{E}-07^{* * *}$ & 15.62 & $8.92 \mathrm{E}-06^{* * *}$ & 12.19 & 8.74E-06*** & 12.21 & $0.029 *$ & 3.59 \\
\hline 2011-SY-DS & 8.66E-07*** & 14.78 & $2.93 \mathrm{E}-04^{* * *}$ & 8.41 & 8.37E-05*** & 9.75 & $0.005^{* *}$ & 5.41 \\
\hline 2012-CP-WW & $7.55 \mathrm{E}-06^{* * *}$ & 12.39 & $0.0037^{* *}$ & 5.74 & $0.0017^{* *}$ & 6.52 & n.s. & - \\
\hline 2012-CP-DS & $7.13 \mathrm{E}-06^{* * *}$ & 12.46 & $0.0159 *$ & 4.21 & $0.0017^{* *}$ & 6.53 & n.s. & - \\
\hline 2012-SY-WW & $7.71 \mathrm{E}-07^{* * *}$ & 14.94 & $1.93 \mathrm{E}-05^{* * *}$ & 11.36 & 5.34E-04*** & 7.77 & n.s. & - \\
\hline 2012-SY-DS & $2.80 \mathrm{E}-06^{* * *}$ & 13.48 & $0.0278^{*}$ & 3.64 & $0.0011^{* *}$ & 7.00 & $0.0134^{*}$ & 4.39 \\
\hline
\end{tabular}

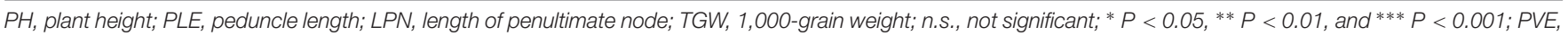

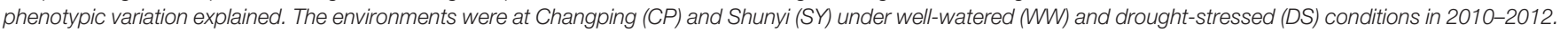



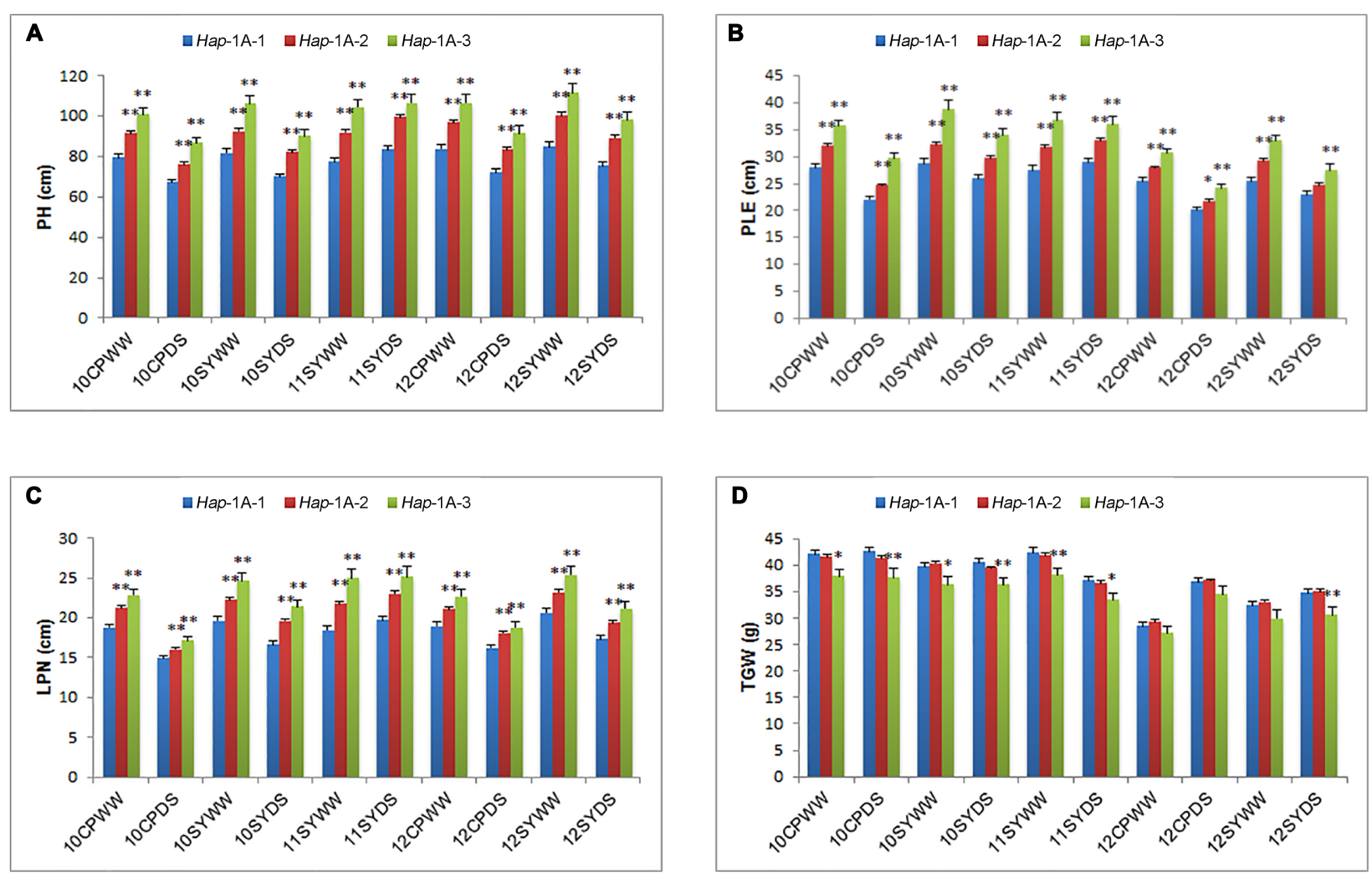

FIGURE 4 | Phenotypic comparisons of three TaSnRK2.3-1A haplotypes in 10 environments. Traits include PH (A), PLE (B), LPN (C), and TGW (D). *, ** indicate significance at $P=0.05$ and 0.01, respectively. Error bars denote SE. See footnote to Table 2 for abbreviations.

environments, and TGW in five environments (Table 3). Hap1B-1 was significantly associated with lower PH, PLE, and LPN, and higher TGW (Figure 5). Therefore, Hap-1B-1 might be a favorable haplotype in terms of $\mathrm{PH}$ and TGW.

\section{Association Analysis between Haplotypes of TaSnRK2.3-1B and SWSC}

For TaSnRK2.3-1B, significant associations were detected between the two haplotypes and SWSC, including WSC1-2-DS, WSC1-3-DS, WSC2-1-DS, WSC2-2-DS/WW, and WSC2-3DS/WW (Table 4). Hap-1B-1 had higher SWSC than Hap-1B-2 at flowering and mid-grain filling stages with significant difference $(P<0.01)$ (Figure 6). So Hap-1B-1 might be a potential haplotype to increase SWSC.

\section{Geographic Distribution of Haplotypes of TaSnRK2.3-1A and TaSnRK2.3-1B in 10 Chinese Wheat Production Zones}

The Chinese wheat production area is classified into 10 main agro-ecological zones based on cultivar ecotypes, growing season, and cultivar response to temperature and photoperiod (Zhang et al., 2002). Among landraces, selection pressure on haplotypes in the different zones was not as strong as expected, and the frequencies of the favored haplotype Hap-1A-1 was generally low, none was identified in zone V, VII, and IX
(Figure 7A). Similar trends were also observed for TaSnRK2.3-1B (Figure 8A).

AS shown in Figures 7, 8, from Chinese landraces to modern cultivars, the frequencies of the two superior haplotypes Hap-1A-1 and Hap-1B-1 increased across almost all zones except in VI (40\% to 33\%) and VIII (36\% to $33 \%$ ) of Hap-1B-1. Hap-1B-1 was the most favorable haplotype in major wheat production regions I (58\%), II (49\%), III (52\%), and IV (83\%) compared to the other six regions in Population 3. The results indicated the two haplotypes had suffered strong positive selection in Chinese wheat breeding programs.

\section{Hap-1A-1 and Hap-1B-1 Were Positively Selected in the Process of Chinese Wheat Breeding}

Three hundred and forty-eight Chinese modern cultivars were divided into six subgroups according to 10 -year release intervals to evaluate changes in haplotype frequencies over time. As a whole, the proportions of the two favored haplotypes, Hap-1A1 and Hap-1B-1, increased gradually with $\mathrm{PH}$ reduction and TGW enhancement in Chinese modern cultivars since pre-1950 (Figure 9), suggesting that these favored haplotypes experienced positive selection in the process of wheat breeding. Furthermore, Hap-1B-1 (up to 75.9\%) was positively selected by wheat breeders, 
TABLE 3 | TaSnRK2.3-1B haplotypes associated with agronomic traits in 10 environments.

\begin{tabular}{|c|c|c|c|c|c|c|c|c|}
\hline \multirow[t]{2}{*}{ Environments } & \multicolumn{2}{|c|}{ PH } & \multicolumn{2}{|c|}{ PLE } & \multicolumn{2}{|c|}{ LPN } & \multicolumn{2}{|c|}{ TGW } \\
\hline & $P$-value & PVE (\%) & $P$-value & PVE (\%) & $P$-value & PVE (\%) & $P$-value & PVE (\%) \\
\hline 2010-CP-WW & $4.83 \mathrm{E}-05^{* * *}$ & 17.10 & $9.39 \mathrm{E}-04^{* * *}$ & 11.21 & $1.98 \mathrm{E}-08^{* * *}$ & 33.65 & $0.0201^{*}$ & 5.48 \\
\hline 2010-CP-DS & $5.32 \mathrm{E}-05^{* * *}$ & 16.90 & 9.23E-04*** & 11.24 & $1.44 \mathrm{E}-05^{* * *}$ & 19.57 & n.s. & - \\
\hline 2010-SY-WW & $1.45 \mathrm{E}-04^{* * *}$ & 14.92 & $0.0013^{* *}$ & 10.62 & $1.06 \mathrm{E}-07^{* * *}$ & 30.14 & n.s. & - \\
\hline 2010-SY-DS & $2.08 \mathrm{E}-04^{* * *}$ & 14.17 & $0.0025^{* *}$ & 9.31 & $9.83 \mathrm{E}-08^{* * *}$ & 30.12 & n.s. & - \\
\hline 2011-SY-WW & 7.16E-04*** & 11.74 & $0.0023^{* *}$ & 9.52 & 1.13E-06*** & 24.90 & $0.0316^{*}$ & 4.67 \\
\hline 2011-SY-DS & $6.84 \mathrm{E}-05^{* * *}$ & 16.40 & $1.04 \mathrm{E}-04^{* * *}$ & 15.57 & $4.98 \mathrm{E}-07^{* * *}$ & 26.65 & n.s. & - \\
\hline 2012-CP-WW & $7.01 \mathrm{E}-05^{* * *}$ & 16.36 & $0.0235^{*}$ & 5.20 & $9.15 \mathrm{E}-05^{* * *}$ & 15.83 & n.s. & - \\
\hline 2012-CP-DS & $3.58 \mathrm{E}-05^{* * *}$ & 17.73 & $0.0021^{* *}$ & 9.67 & $4.15 \mathrm{E}-08^{* * *}$ & 32.09 & $0.0171^{*}$ & 5.76 \\
\hline 2012-SY-WW & $1.36 \mathrm{E}-05^{* * *}$ & 19.73 & $0.0018^{* *}$ & 9.97 & $1.51 \mathrm{E}-07^{* * *}$ & 29.25 & $0.0039 * *$ & 8.49 \\
\hline 2012-SY-DS & $2.95 \mathrm{E}-04^{* * *}$ & 13.49 & $0.0279 *$ & 4.89 & $2.31 \mathrm{E}-05^{* * *}$ & 18.62 & 7.19E-04*** & 11.73 \\
\hline
\end{tabular}

$\overline{P H}$, plant height; PLE, peduncle length; LPN, length of penultimate node; TGW, 1,000-grain weight; n.s., not significant; $* P<0.05, * * P<0.01$, and $* * * P<0.001 ; P V E$,

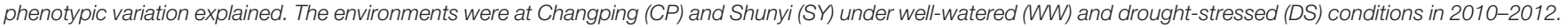
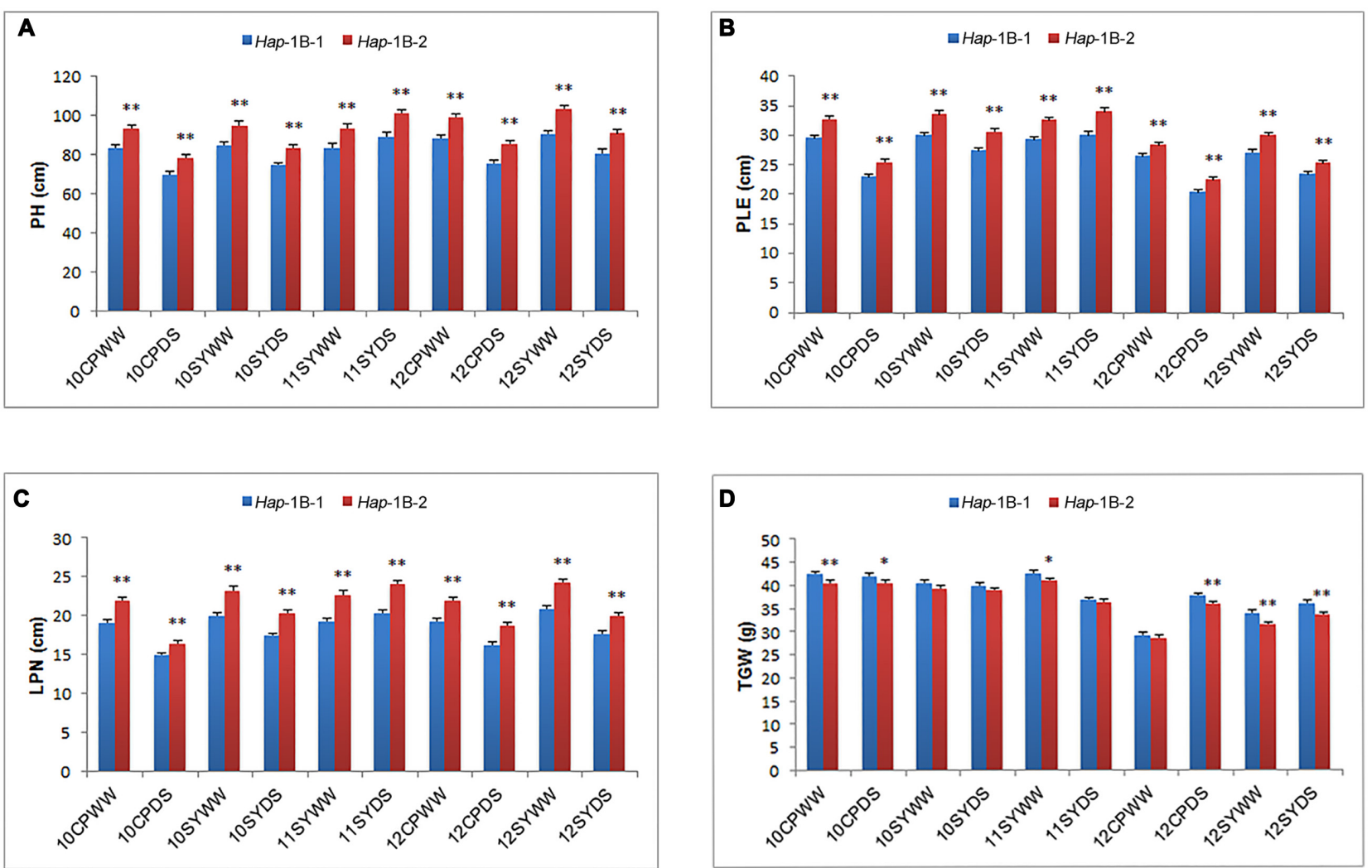

FIGURE 5 | Phenotypic comparisons of two TaSnRK2.3-1B haplotypes in 10 environments. Traits include PH (A), PLE (B), LPN (C), and TGW (D). *, ** indicate significance at $P=0.05$ and 0.01 , respectively. Error bars denote SE. See footnote to Table $\mathbf{3}$ for abbreviations.

while Hap-1A-1 (only 55.9\%) still has considerable potential for further utilization.

\section{DISCUSSION}

In this study, we obtained the three genomic sequences of TaSnRK2.3-1A, TaSnRK2.3-1B, and TaSnRK2.3-1D; however, there was no polymorphism identified in TaSnRK2.3-1D, therefore, TaSnRK2.3-1D was not included for polymorphism analysis. DNA polymorphism analysis results revealed that the variations for TaSnRK2.3-1A only occurred in non-exon

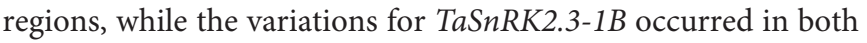
introns and exons. Further association analysis indicated both TaSnRK2.3-1A and TaSnRK2.3-1B were significantly associated with PH, PLE, LPN, and TGW, thus we speculate that the two 
TABLE 4 | TaSnRK2.3-1B haplotypes associated with stem water-soluble carbohydrates (SWSC) at different grain-filling stages.

\begin{tabular}{|c|c|c|c|c|c|c|c|c|}
\hline Traits & $P$-Value & PVE (\%) & Traits & $P$-Value & PVE (\%) & Traits & $P$-Value & PVE (\%) \\
\hline WSC1-1-DS & n.s. & - & WSC2-1-DS & $0.0016^{* *}$ & 10.25 & WSC3-1-DS & n.s. & - \\
\hline WSC1-2-DS & $5.82 \mathrm{E}-07^{* * *}$ & 26.36 & WSC2-2-DS & $0.0012^{* *}$ & 10.83 & WSC3-2-DS & n.s. & - \\
\hline WSC1-3-DS & $2.65 \mathrm{E}-04^{* * *}$ & 13.69 & WSC2-3-DS & $0.0013^{* *}$ & 10.59 & WSC3-3-DS & n.s. & - \\
\hline WSC1-1-WW & n.s. & - & WSC2-1-WW & n.s. & - & WSC3-1-WW & n.s. & - \\
\hline WSC1-2-WW & n.s. & - & WSC2-2-WW & $0.0030 * *$ & 8.97 & WSC3-2-WW & n.s. & - \\
\hline WSC1-3-WW & n.s. & - & WSC2-3-WW & $0.0028 * *$ & 9.12 & WSC3-3-WW & n.s. & - \\
\hline
\end{tabular}

WSC1-1-DS/WW, WSC1-2-DS/WW, and WSC1-3-DS/WW, WSC content of peduncle, lower stem internode and total stem under drought-stressed/well-watered at flowering stage, respectively. WSC2-1-DS/WW, WSC2-2-DS/WW, and WSC2-3-DS/WW, WSC content of peduncle, lower stem internode and total stem under droughtstressed/well-watered at mid-grain filling stage, respectively. WSC3-1-DS/WW, WSC3-2-DS/WW, and WSC3-3-DS/WW indicate WSC content of peduncle, lower stem internode and total stem under drought-stressed/well-watered at maturity stage. n.s., not significant; ** $P<0.01$ and ${ }^{* * *} P<0.001$; PVE, phenotypic variation explained.

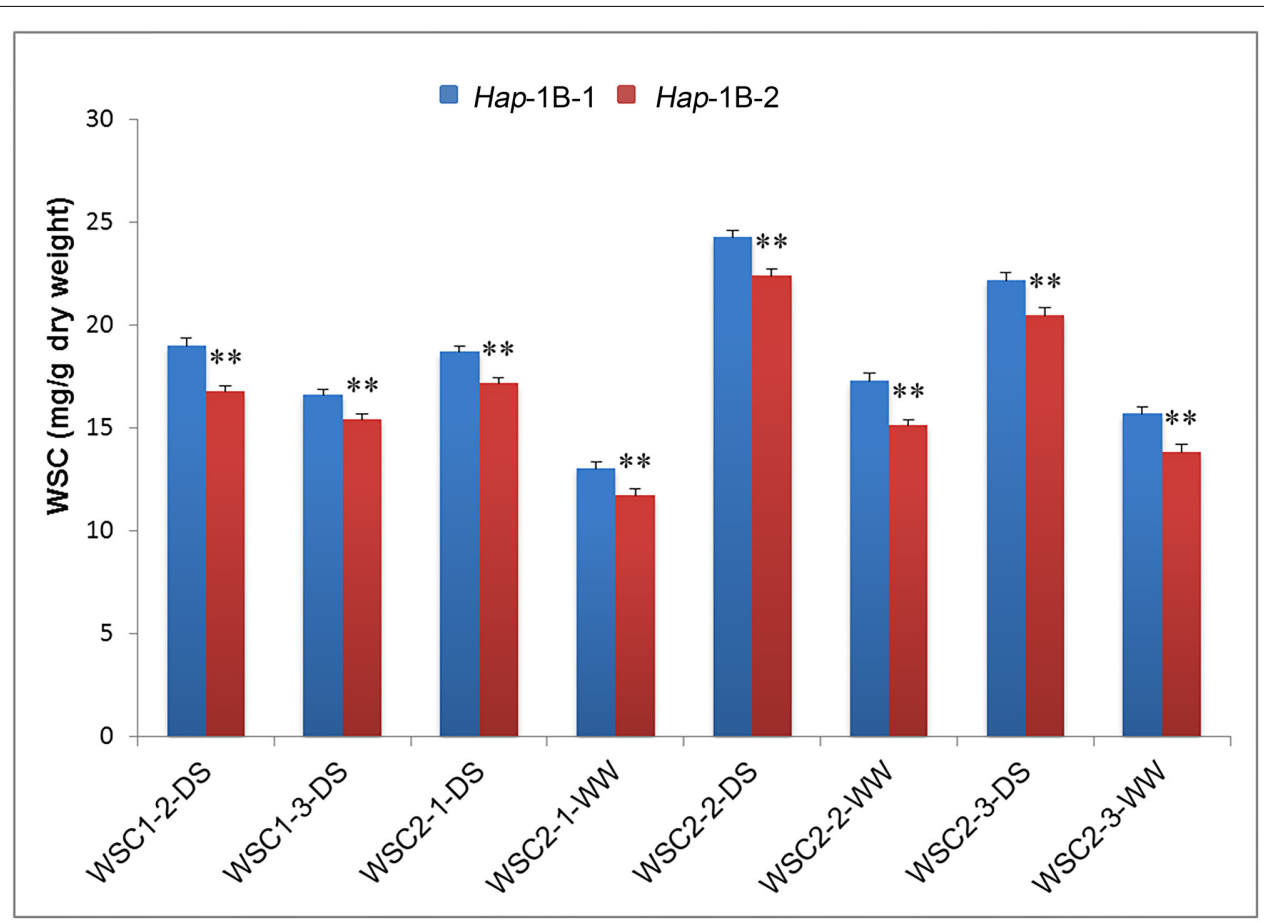

FIGURE 6 | Comparison of stem water-soluble carbohydrates (SWSC) associated with haplotypes of TaSnRK2.3-1B at different grain filling stages. ** indicates significance at $P=0.01$. Error bars denote SE. See footnote to Table 4 for description of traits.
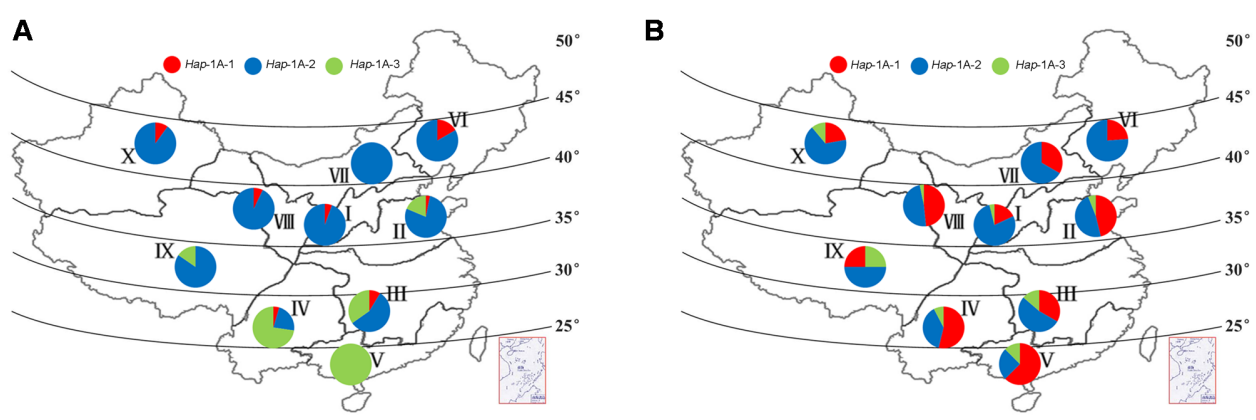

FIGURE 7 | Haplotype distribution of TaSnRK2.3-1A in $\mathbf{1 0}$ Chinese wheat ecological regions. (A) Distribution of TaSnRK2.3-1A haplotypes in 157 Chinese landraces. (B) Distribution of TaSnRK2.3-1A haplotypes in 348 modern cultivars. I, Northern winter wheat region; II, Yellow and Huai River valley winter wheat region; III, Low and middle Yangtze River valley winter wheat region; IV, Southwestern winter wheat region; V, Southern winter wheat region; VI, Northeastern spring wheat region; VII, Northern spring wheat region; VIII, Northwestern spring wheat region; IX, Qinghai-Tibet spring-winter wheat region; X, Xinjiang winter-spring wheat region. 

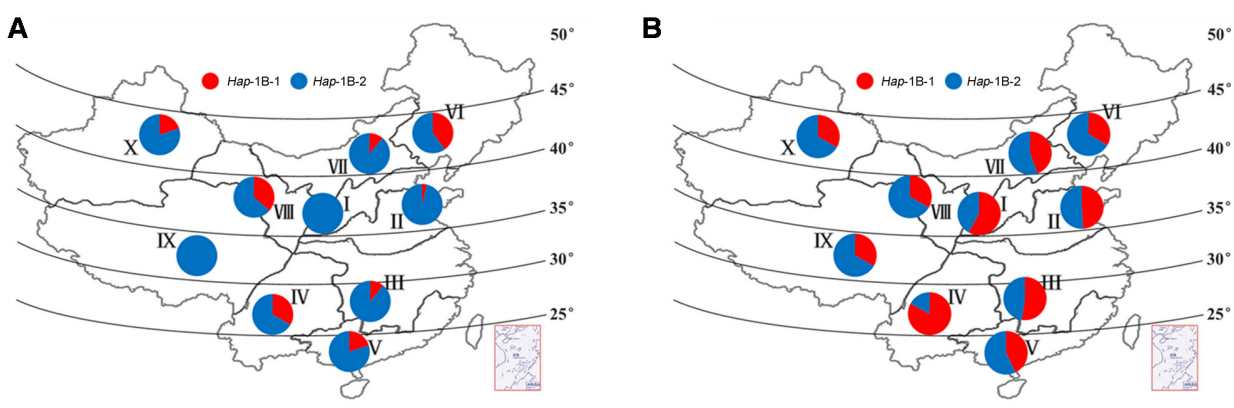

FIGURE 8 | Distribution of TaSnRK2.3-1B haplotypes in 10 Chinese wheat ecological regions. (A) Distribution of TaSnRK2.3-1B haplotypes in 157 Chinese landraces. (B) Distribution of TaSnRK2.3-1B haplotypes in 348 modern cultivars. See footnote to Figure $\mathbf{7}$ for abbreviations from I to X.
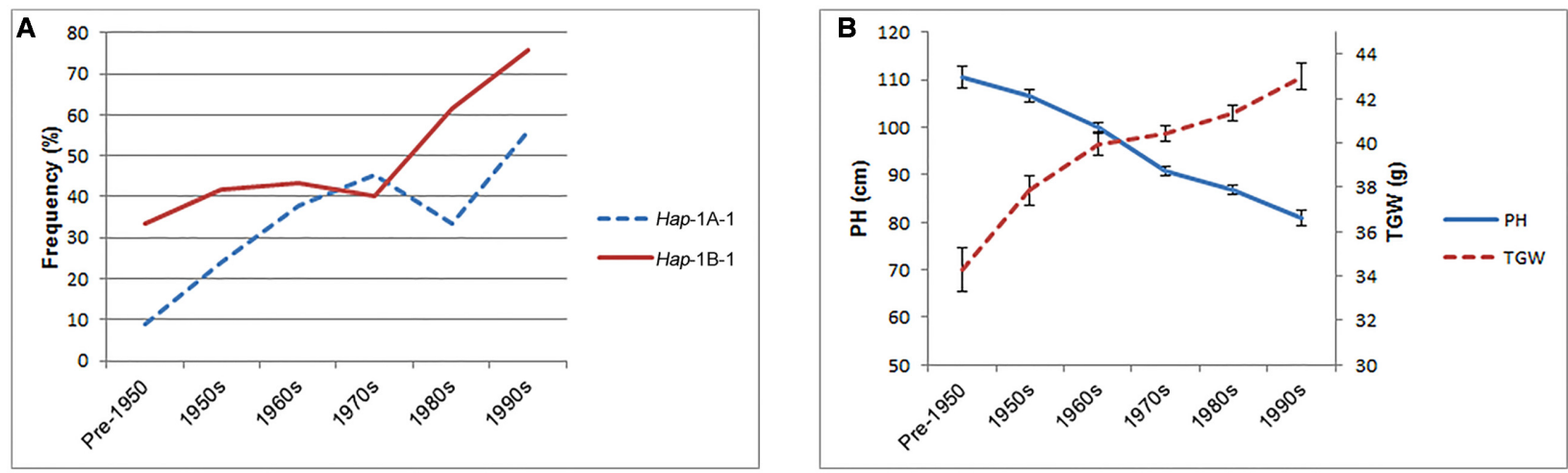

FIGURE 9 | Elite haplotypes Hap-1A-1 and Hap-1B-1 were positively selected in Chinese wheat breeding programs. (A) The frequencies of two favored haplotype in Population 3 over decades. 12, 25, 55, 102, 106, and 34 accessions were released in the pre-1950, 1950s, 1960s, 1970s, 1980s, and 1990s, respectively. Fourteen accessions with unknown release dates were excluded. (B) The changes of PH and TGW in Population 3 over decades. Error bars denote $2 \times$ SE.

genes might have similar functions. Further studies need to be performed to validate the allelic effects of these polymorphisms, such as gene expression analysis.

Stem water-soluble carbohydrates are not only a main source for grain filling, but also crucial osmolytes in regulating cell turgor under abiotic stress conditions in wheat (Yang et al., 2007). Association analysis results showed that TaSnRK2.3-1B was associated with SWSC under three WW conditions and five DS conditions, which was similar to previous studies of TaSnRK2.7$B$ and TaSnRK2.8-A in SWSC metabolism (Zhang et al., 2011a,b, 2013). Since TaSnRK2.3, TaSnRK2.7, and TaSnRK2.8 belong to SnRK2 subfamily, they might function similarly in carbohydrate metabolism. Moreover, SWSC under DS conditions were clearly higher than SWSC under corresponding WW conditions, which agrees with the observation that drought induced WSC remobilization increases in response to water deficit (Goggin and Setter, 2004; Rebetzke et al., 2008).

Compelling evidence demonstrates that yeast SNF1-kinase and mammalian AMPK, and plant SnRK1 participate in sugar metabolism, including starch synthesis and carbohydrate distribution (Halford et al., 2003; Schwachtje et al., 2006). Further data support that SnRK2 and SnRK3 originated by duplication of SnRK1 and then diverged rapidly during plants response to adverse stresses (Hrabak et al., 2003; Hauser et al., 2011). However, our results support that TaSnRK2.3 is involved in both abiotic stress responses and SWSC metabolism, suggesting it still maintains ancient functions, which was also observed in TaSnRK2.7 and TaSnRK2.8 (Zhang et al., 2011a).

For TaSnRK2.3-1B, the favored haplotype Hap-1B-1 had the higher TGW and higher SWSC at flowering and mid-grain filling stages, consistent with earlier results that there were significant correlations between TGW and SWSC (Zhang et al., 2014; Li et al., 2015). Li et al. (2015) indicated that SWSC can make a positive contribution to TGW under variable water conditions. High SWSC were suggested as an useful trait for improving grain weight in wheat breeding (Shearman et al., 2005; Ruuska et al., 2006). High grain yield is one of the most important breeding objectives in wheat improvement. The high heritability values of TGW have proved it is phenotypically the most stable yield component which continuously attracts the attention of breeders.

In our previous study, TaSnRK2.3-1B was mapped to a region flanked by wmc156 $(2.1 \mathrm{cM})$ and $p 3446-183(2.9 \mathrm{cM})$ on chromosome $1 \mathrm{~B}$ in the $\mathrm{DH}$ population derived from a cross of Hanxuan $10 \times$ Lumai 14, co-located with a quantitative trait locus controlling PH (Wu et al., 2010, 2012; Tian et al., 2013). 
Here, our association data demonstrated that TaSnRK2.3-1B was significantly associated with $\mathrm{PH}$, and the favored haplotypes had lower PH. Additionally, as shown in Figure 9B, PH decreased in stepwise manner over decades and was positively selected in the process of Chinese wheat breeding. Therefore, we speculate that $T a S n R K 2.3-1 B$ might be a potential gene related to $\mathrm{PH}$ or closely linked with genes involved in $\mathrm{PH}$ regulation.

Among various markers, functional markers derived from polymorphic sites within target genes, are superior to conventional molecular markers such as RFLPs, SSRs, and AFLPs because of complete linkage with trait locus alleles, and are ideal for marker-assisted breeding. In the current study, functional markers were developed for genotyping based on variants in TaSnRK2.3 genes. In sum, the CAPS/dCAPS markers 2.3AM1, 2.3AM2, 2.3BM1, and 2.3BM2 were used successfully. Combinations of the two markers 2.3AM1 and 2.3AM2 for A genome formed three haplotypes that significantly affected important agronomic traits, similarly to other two markers 2.3BM1 and 2.3BM2 in B genome. These four markers are all co-dominant and allow efficient assays of large DNA samples in a simple, rapid, and low-cost procedure, which is performed in most molecular biology and/or plant breeding laboratories.

To sum up, the two elite haplotypes Hap-1A-1 and Hap1B-1 of TaSnRK2.3s, can contribute positively to grain size

\section{REFERENCES}

Agrama, H. A., Eizenga, G. C., and Yan, W. (2007). Association mapping of yield and its components in rice cultivars. Mol. Breed. 19, 341-356. doi: 10.1007/ s11032-006-9066-6

Collard, B. C., and Mackill, D. J. (2008). Marker-assisted selection: an approach for precision plant breeding in the twenty-first century. Philos. Trans. $R$ Soc. Lond. B Biol. Sci. 363, 557-572. doi: 10.1098/rstb.2007.2170

Dong, Y., Cao, Y., Zhang, X., Liu, S., Wang, L., You, G., et al. (2003). Establishment of candidate core collections in Chinese common wheat germplasm. J. Plant Genet. Resour. 4, 1-8. doi: 10.13430/j.cnki.jpgr.2003.01.001

Fujii, H., Verslues, P. E., and Zhu, J. (2011). Arabidopsis decuple mutant reveals the importance of SnRK2 kinases in osmotic stress responses in vivo. Proc. Natl. Acad. Sci. U.S.A. 108, 1717-1722. doi: 10.1073/pnas.1018367108

Goggin, D. E., and Setter, T. L. (2004). Fructosyltransferase activity and fructan accumulation during development in wheat exposed to terminal drought. Funct. Plant Biol. 31, 11-21. doi: 10.1071/FP03123

Halford, N. G., Hey, S., Jhurreea, D., Laurie, S., McKibbin, R. S., Paul, M., et al. (2003). Metabolic signalling and carbon partitioning: role of Snf1-related (SnRK1) protein kinase. J. Exp. Bot. 54, 467-475. doi: 10.1093/jxb/erg038

Hao, C., Dong, Y., Wang, L., You, G., Zhang, H., Ge, H., et al. (2008). Genetic diversity and construction of core collection in Chinese wheat genetic resources. Chin. Sci. Bull. 53, 1518-1526. doi: 10.1007/s11434-008-0212-x

Hao, C., Wang, L., Ge, H., Dong, Y., and Zhang, X. (2011). Genetic diversity and linkage disequilibrium in Chinese bread wheat (Triticum aestivum L.) revealed by SSR markers. PLoS ONE 6:e17279. doi: 10.1371/journal.pone.0017279

Hauser, F., Waadt, R., and Schroeder, J. I. (2011). Evolution of abscisic acid synthesis and signaling mechanisms. Curr. Biol. 21, R346-R355. doi: 10.1016/j. cub.2011.03.015

Hong, S. W., Jon, J. H., Kwak, J. M., and Nam, H. G. (1997). Identification of a receptor-like protein kinase gene rapidly induced by abscisic acid, dehydration, high salt, and cold treatments in Arabidopsis thaliana. Plant Physiol. 113, 1203-1212. doi: 10.1104/pp.113.4.1203

Hrabak, E. M., Chan, C. W., Gribskov, M., Harper, J. F., Choi, J. H., Halford, N., et al. (2003). The Arabidopsis CDPK-SnRK superfamily of protein kinases. Plant Physiol. 132, 666-680. doi: 10.1104/pp.102.011999 enhancement and $\mathrm{PH}$ reduction in wheat. They could be applied in wheat breeding programs using marker assisted selection.

\section{AUTHOR CONTRIBUTIONS}

Conceived the idea: RJ, XM, LM, and ZW. Performed the experiments: LM, XM, JW, ZL, BZ, WL, and XC. Analyzed the data: LM, XM, and RJ. Wrote the manuscript: LM and XM. Revised the manuscript: RJ, XM, and MR.

\section{FUNDING}

This work was supported by the National Natural Science Foundation of China (31571660, 31461143024), and Agricultural Science and Technology Innovation Program (ASTIP).

\section{ACKNOWLEDGMENTS}

We thank our colleagues Dr. Xueyong Zhang and Dr. Chenyang Hao (Institute of Crop Science, CAAS) for providing DNA samples of Population 2 (157 landraces) and Population 3 (348 modern cultivars).

Huang, J., Teyton, L., and Harper, J. F. (1996). Activation of a $\mathrm{Ca}^{2+}$-dependent protein kinase involves intramolecular binding of a calmodulin-like regulatory domain. Biochemistry 35, 13222-13230. doi: 10.1021/bi960498a

International Wheat Genome Sequencing Consortium [IWGSC] (2014). A chromosome-based draft sequence of the hexaploid bread wheat (Triticum aestivum) genome. Science 345:1251788. doi: 10.1126/science.1251788

Jia, J., Zhao, S., Kong, X., Li, Y., Zhao, G., He, W., et al. (2013). Aegilops tauschii draft genome sequence reveals a gene repertoire for wheat adaptation. Nature 496, 91-95. doi: 10.1038/nature 12028

Kobayashi, Y., Yamamoto, S., Minami, H., Kagaya, Y., and Hattori, T. (2004). Differential activation of the rice sucrose nonfermenting1-related protein kinase 2 family by hyperosmotic stress and abscisic acid. Plant Cell 16, 1163-1177. doi: 10.1105/tpc.019943

Kulik, A., Wawer, I., Krzywinska, E., Bucholc, M., and Dobrowolska, G. (2011). SnRK2 protein kinases-key regulators of plant response to abiotic stresses. OMICS 15, 859-872. doi: 10.1089/omi.2011.0091

Li, B., Li, Q., Mao, X., Li, A., Wang, J., Chang, X., et al. (2016). Two novel AP2/EREBP transcription factor genes TaPARG have pleiotropic functions on plant architecture and yield-related traits in common wheat. Front. Plant Sci. 7:1191. doi: 10.3389/fpls.2016.01191

Li, Q., Li, L., Yang, X., Warburton, M. L., Bai, G., Dai, J., et al. (2010). Relationship, evolutionary fate and function of two maize co-orthologs of rice GW2 associated with kernel size and weight. BMC Plant Biol. 10:143. doi: 10.1186/ 1471-2229-10-143

Li, W., Zhang, B., Li, R., Chang, X., and Jing, R. (2015). Favorable alleles for stem water-soluble carbohydrates identified by association analysis contribute to grain weight under drought stress conditions in wheat. PLoS ONE 10:e0119438. doi: 10.1371/journal.pone.0119438

Ling, H., Zhao, S., Liu, D., Wang, J., Sun, H., Zhang, C., et al. (2013). Draft genome of the wheat A-genome progenitor Triticum urartu. Nature 496, 87-90. doi: 10.1038/nature11997

Ludwig, A. A., Romeis, T., and Jones, J. D. (2004). CDPK-mediated signalling pathways: specificity and cross-talk. J. Exp. Bot. 55, 181-188. doi: 10.1093/jxb/ erh008

Nemri, A., Atwell, S., Tarone, A. M., Huang, Y. S., Zhao, K., Studholme, D. J., et al. (2010). Genome-wide survey of Arabidopsis natural variation in downy mildew 
resistance using combined association and linkage mapping. Proc. Natl. Acad. Sci. U.S.A. 107, 10302-10307. doi: 10.1073/pnas.0913160107

Rebetzke, G. J., Van Herwaarden, A. F., Jenkins, C., Weiss, M., Lewis, D., Ruuska, S., et al. (2008). Quantitative trait loci for water-soluble carbohydrates and associations with agronomic traits in wheat. Aust. J. Agric. Res. 59, 891-905. doi: 10.1071/AR08067

Ruuska, S. A., Rebetzke, G. J., Herwaarden, A. F. V., Richards, R. A., Fettell, N. A., Tabe, L., et al. (2006). Genotypic variation in water-soluble carbohydrate accumulation in wheat. Funct. Plant Biol. 33, 799-809. doi: 10.1071/FP 06062

Schwachtje, J., Minchin, P. E., Jahnke, S., van Dongen, J. T., Schittko, U., and Baldwin, I. T. (2006). SNF1-related kinases allow plants to tolerate herbivory by allocating carbon to roots. Proc. Natl. Acad. Sci. U.S.A. 103, 12935-12940. doi: $10.1073 /$ pnas.0602316103

Shearman, V. J., Sylvesterbradley, R., Scott, R. K., and Foulkes, M. J. (2005). Physiological processes associated with wheat yield progress in the UK. Crop Sci. 45, 175-185. doi: 10.2135/cropsci2005.0175

Stewart, C. N. Jr., and Via, L. E. (1993). A rapid CTAB DNA isolation technique useful for RAPD fingerprinting and other PCR applications. Biotechniques 14, $748-750$.

Thornsberry, J. M., Goodman, M. M., Doebley, J., Kresovich, S., Nielsen, D., and Buckler, E. S. T. (2001). Dwarf8 polymorphisms associate with variation in flowering time. Nat. Genet. 28, 286-289. doi: 10.1038/90135

Tian, S., Mao, X., Zhang, H., Chen, S., Zhai, C., Yang, S., et al. (2013). Cloning and characterization of TaSnRK2.3, a novel SnRK2 gene in common wheat. J Exp Bot 64, 2063-2080. doi: 10.1093/jxb/ert072

Varshney, R. K., Hoisington, D. A., and Tyagi, A. K. (2006). Advances in cereal genomics and applications in crop breeding. Trends Biotechnol. 24, 490-499. doi: 10.1016/j.tibtech.2006.08.006

Vlad, F., Rubio, S., Rodrigues, A., Sirichandra, C., Belin, C., Robert, N., et al. (2009). Protein phosphatases $2 \mathrm{C}$ regulate the activation of the Snf1-related kinase OST1 by abscisic acid in Arabidopsis. Plant Cell 21, 3170-3184. doi: 10.1105/tpc.109. 069179

Wang, B., Tan, H. W., Fang, W., Meinhardt, L. W., Mischke, S., Matsumoto, T., et al. (2015). Developing single nucleotide polymorphism (SNP) markers from transcriptome sequences for identification of longan (Dimocarpus longan) germplasm. Hortic. Res. 2:14065. doi: 10.1038/hortres. 2014.65

Wang, X., Wang, H., Liu, S., Ferjani, A., Li, J., Yan, J., et al. (2016). Genetic variation in ZmVPP1 contributes to drought tolerance in maize seedlings. Nat. Genet. 48, 1233-1241. doi: 10.1038/ng.3636

Wang, Z., Liu, X., Li, R., Chang, X., and Jing, R. (2011). Development of nearinfrared reflectance spectroscopy models for quantitative determination of water-soluble carbohydrate content in wheat stem and glume. Anal. Lett. 44, 2478-2490. doi: 10.1080/00032719.2011.551859

Wrzaczek, M., and Hirt, H. (2001). Plant MAP kinase pathways: how many and what for? Biol. Cell 93, 81-87. doi: 10.1016/S0248-4900(01)01121-2
Wu, X., Chang, X., and Jing, R. (2012). Genetic insight into yield-associated traits of wheat grown in multiple rain-fed environments. PLOS ONE 7:e31249. doi: 10.1371/journal.pone.0031249

Wu, X., Wang, Z., Chang, X., and Jing, R. (2010). Genetic dissection of the developmental behaviours of plant height in wheat under diverse water regimes. J. Exp. Bot. 61, 2923-2937. doi: 10.1093/jxb/erq117

Yang, D., Jing, R., Chang, X., and Li, W. (2007). Identification of quantitativetrait loci and environmental interactions for accumulation and remobilization of water-soluble carbohydrates in wheat (Triticum aestivum L.) stems. Genetics 176, 571-584. doi: 10.1534/genetics.106.068361

Zhang, B., Li, W., Chang, X., Li, R., and Jing, R. (2014). Effects of favorable alleles for water-soluble carbohydrates at grain filling on grain weight under drought and heat stresses in wheat. PLoS ONE 9:e102917. doi: 10.1371/journal.pone. 0102917

Zhang, B., Liu, X., Xu, W., Chang, J., Li, A., Mao, X., et al. (2015). Novel function of a putative MOC1 ortholog associated with spikelet number per spike in common wheat. Sci. Rep. 5:12211. doi: 10.1038/srep12211

Zhang, H., Li, W., Mao, X., Jing, R., and Jia, H. (2016). Differential activation of the wheat SnRK2 family by abiotic stresses. Front. Plant Sci. 7:420. doi: $10.3389 /$ fpls.2016.00420

Zhang, H., Mao, X., and Jing, R. (2011a). SnRK2 acts within an intricate network that links sucrose metabolic and stress signaling in wheat. Plant Signal. Behav. 6, 652-654. doi: 10.4161/psb.6.5.14945

Zhang, H., Mao, X., Wu, X., Wang, C., and Jing, R. (2011b). An abiotic stress response gene TaSnRK2.7-B in wheat accessions: genetic diversity analysis and gene mapping based on SNPs. Gene 478, 28-34. doi: 10.1016/j.gene.2011.01.011

Zhang, H., Mao, X., Zhang, J., Chang, X., and Jing, R. (2013). Single-nucleotide polymorphisms and association analysis of drought-resistance gene TaSnRK2.8 in common wheat. Plant Physiol. Biochem. 70, 174-181. doi: 10.1016/j.plaphy. 2013.04.010

Zhang, X., Li, C., Wang, L., Wang, H., You, G., and Dong, Y. (2002). An estimation of the minimum number of SSR alleles needed to reveal genetic relationships in wheat varieties. I. Information from large-scale planted varieties and cornerstone breeding parents in Chinese wheat improvement and production. Theor. Appl. Genet. 106, 112-117. doi: 10.1007/s00122-002-1016-z

Conflict of Interest Statement: The authors declare that the research was conducted in the absence of any commercial or financial relationships that could be construed as a potential conflict of interest.

Copyright (C) 2017 Miao, Mao, Wang, Liu, Zhang, Li, Chang, Reynolds, Wang and Jing. This is an open-access article distributed under the terms of the Creative Commons Attribution License (CC BY). The use, distribution or reproduction in other forums is permitted, provided the original author(s) or licensor are credited and that the original publication in this journal is cited, in accordance with accepted academic practice. No use, distribution or reproduction is permitted which does not comply with these terms. 